\title{
Frailty and post-operative outcomes in older surgical patients: a systematic review
}

\author{
Hui-Shan Lin ${ }^{*} \mathbb{D}$, J. N. Watts, N. M. Peel and R. E. Hubbard
}

\begin{abstract}
Background: As the population ages, increasing numbers of older adults are undergoing surgery. Frailty is prevalent in older adults and may be a better predictor of post-operative morbidity and mortality than chronological age. The aim of this review was to examine the impact of frailty on adverse outcomes in the 'older old' and 'oldest old' surgical patients.

Methods: A systematic review was undertaken. Electronic databases from 2010 to 2015 were searched to identify articles which evaluated the relationship between frailty and post-operative outcomes in surgical populations with a mean age of 75 and older. Articles were excluded if they were in non-English languages or if frailty was measured using a single marker only. Demographic data, type of surgery performed, frailty measure and impact of frailty on adverse outcomes were extracted from the selected studies. Quality of the studies and risk of bias was assessed by the Epidemiological Appraisal Instrument.

Results: Twenty-three studies were selected for the review and they were assessed as medium to high quality. The mean age ranged from 75 to 87 years, and included patients undergoing cardiac, oncological, general, vascular and hip fracture surgeries. There were 21 different instruments used to measure frailty. Regardless of how frailty was measured, the strongest evidence in terms of numbers of studies, consistency of results and study quality was for associations between frailty and increased mortality at 30 days, 90 days and one year follow-up, post-operative complications and length of stay. A small number of studies reported on discharge to institutional care, functional decline and lower quality of life after surgery, and also found a significant association with frailty.

Conclusion: There was strong evidence that frailty in older-old and oldest-old surgical patients predicts postoperative mortality, complications, and prolonged length of stay. Frailty assessment may be a valuable tool in perioperative assessment. It is possible that different frailty tools are best suited for different acuity and type of surgical patients. The association between frailty and return to pre-morbid function, discharge destination, and quality of life after surgery warrants further research.
\end{abstract}

Keywords: Post-operative complications, Mortality, Geriatric, Oldest old, Frailty

Abbreviations: FI, Frailty index; CSHA, Canadian Study of Health and Aging; EAl, Epidemiological appraisal instrument; CGA, Comprehensive geriatric assessment; MACCE, Major cardiac and cerebral adverse events

\footnotetext{
*Correspondence: huishan.lin@uq.net.au

Centre for Research in Geriatric Medicine, Princess Alexandra Hospital, The

University of Queensland, Level 2, Building 33, Ipswich Road,

Woolloongabba, QLD 4102, Australia
}

(c) 2016 The Author(s). Open Access This article is distributed under the terms of the Creative Commons Attribution 4.0 International License (http://creativecommons.org/licenses/by/4.0/), which permits unrestricted use, distribution, and reproduction in any medium, provided you give appropriate credit to the original author(s) and the source, provide a link to the Creative Commons license, and indicate if changes were made. The Creative Commons Public Domain Dedication waiver (http://creativecommons.org/publicdomain/zero/1.0/) applies to the data made available in this article, unless otherwise stated. 


\section{Background}

As the population ages, the rate of surgical procedures in the older population is rising. In England, 2.5 million people over the age of 75 years underwent surgery between years 2014 and 2015, as opposed to just under 1.5 million between 2006 and 2007 [1, 2]. Nearly $30 \%$ of these 2.5 million were over 85 years old [1]. Similarly, women aged 85 years and over now represent the largest proportion in emergency surgical admissions in Australia compared with all other age and sex groups [3].

It has long been recognised that advanced age can carry increased risk of mortality and morbidity after surgery. However, new knowledge is emerging that frailty, an age-related cumulative decline in multiple physiological systems, is a better predictor of mortality and morbidity than chronological age $[4,5]$. Patients of the same age do not all have the same risk. The identification and assessment of frailty may facilitate identification of vulnerable surgical patients so that appropriate surgical and anaesthetic management can be implemented.

Experienced clinicians may feel that they can identify frailty by end-of-bed 'gestalt' assessments. However, 'eyeballing' is subjective and tends to be inconsistent between different observers [6]. Currently there is no standardised method of measuring frailty, with more than 20 different frailty instruments identified in a systematic review [7]. These different scales are based on the two main models which characterise how frailty develops and manifests. In the 'phenotype' model described by Fried et al. [8], frailty manifests as decline in lean body mass, strength, endurance, balance, walking performance and low activity. Patients who have three or more of the five features of slowness, weakness, exhaustion, weight loss and low physical activity are deemed frail, while those who have none of the features are nonfrail. Patients who display one or two of the five features are "pre-frail" [8].

The second model by Rockwood et al. is the Frailty Index (FI), or the cumulative deficit model, developed in the Canadian Study of Health and Aging (CSHA) [9]. This model conceptualises aging as the accumulation of deficits and views frailty as a multidimensional risk state quantified by the number of deficits rather than by the nature of the health problems. An FI can be based on comprehensive geriatric assessment and is calculated by counting the number of deficits present in an individual, divided by the total number of deficits measured [10]. The deficits encompass co-morbidities, physical and cognitive impairments, psychosocial risk factors and common geriatric syndromes [10]. The FI score ranges between 0 and 1 , with higher scores indicating greater degree of frailty. FI represents a continuum; however, it can also be trichotomised to indicate low, intermediate and high level of frailty ( $\mathrm{FI} \leq 0.25, \mathrm{FI}>0.25-0.4$, FI $>0.4$ ) [11].
There has been a significant increase in literature over the last five years on the subject of frailty in surgical patients. A search for articles on Pubmed published between the years 2011 and 2015 using search terms 'frailty' AND 'surgical outcome' identified 173 titles, whereas the same search for publications between 2006 and 2010 yielded only 34 titles. The majority of the current literature investigating frailty and surgery has defined 'geriatric' as those above 60 or 65 years old. However, there has been a change in who is thought of as 'old'. Basing studies on someone 65 years old may not provide insight into appropriate treatment for the 'new' geriatric patient [12]. Despite frailty being more prevalent with increasing age, and the large proportion of those over 75 years old undergoing surgery, frailty in the 'old old' and the 'oldest old' (aged 75-85 and over 85 years) surgical patients has been less comprehensively explored.

The aim of this systematic review, therefore, was to examine the association between frailty and adverse post-surgical outcomes in patients aged 75 years and over.

\section{Methods}

\section{Search strategy}

PUBMED, MEDLINE, EMBASE and Cochrane online databases were searched using search terms of 'frail"' AND 'surg"' in combination with ('outcome' OR 'morbidity' OR 'complication'). An asterisk was used to indicate the term was truncated or had a variation in spelling. The search was conducted between October and December 2015 with filters applied to limit results to the English language, human research, and publications from year 2010 and onwards.

\section{Publication selection}

The inclusion criteria for the search were: 1) the mean participant age was over 75 years; 2) the patient population had a surgical procedure; 3 ) frailty was assessed as a composite measure of more than one domain of health deficit, which accords with the current conceptualisation of frailty $[13,14]$ and was the main factor of interest in the study; and 4) the relationship between frailty and adverse outcomes was evaluated. Exclusion criteria were review articles, conference abstracts, and studies which measured frailty as a single item, such as a scan finding, a blood marker, or a physical performance test such as gait speed.

\section{Data extraction}

Two reviewers (HL, JW) conducted the searches independently and compared results after assessing all identified abstracts for their compliance with the review criteria. Where agreement could not be reached a third 
independent reviewer (NP) was consulted. Reasons for exclusion were documented.

The following data were extracted from the eligible studies: sample size, mean age, country of origin of the study population, study design, type of surgery performed, frailty measure, and impact of frailty on adverse outcome.

\section{Assessment of study quality and risk of bias}

Two reviewers (HL, JW) independently assessed the quality of the included studies using a modified version of the Epidemiological Appraisal Instrument (EAI), a valid and reliable tool for rating the quality of observational studies [15]. The EAI checklist addressed the following five domains of risk of bias: reporting, subject selection, measurement quality, data analysis, and generalisation of results. Each of the 23 questions in the EAI applicable to the selected studies was scored as yes $(=2)$, partial $(=1)$, no or unable to determine $(=0)$ with the highest possible score being 46 .

An a priori decision was made to divide the total possible score into quartiles. Quartile 1 (Q1) was 35-46 (the highest quality), quartile $2(\mathrm{Q} 2)$ was $23-34$, quartile 3 (Q3) was 12-23 and quartile 4 (Q4) was 0-11 (the lowest quality). Any disagreement regarding the assessment of the quality of a study was resolved by consulting a third reviewer (NP).

\section{Grading the overall strength of the evidence}

The overall strength of the evidence was evaluated using principles outlined by the Agency for Healthcare Research and Quality [16]. The key elements of evaluation were quality (based on study design according to the hierarchy of evidence and study execution), quantity (based on the number of studies) and consistency.

\section{Results}

The literature search identified 686 articles (187 from Pubmed, 169 from Medline, 300 from Embase and 28 from the Cochrane database). From these, 270 duplicate articles were removed. The titles, abstracts and the full texts of the articles were reviewed. Articles were selected based on inclusion and exclusion criteria. The references of selected articles were hand searched for further eligible articles. There were 23 articles included in the final analysis. The study selection process as well as the reasons for exclusion are shown in Fig. 1.

In the 23 articles selected for this review, there were 16 cohorts of patients with a mean or median age ranging from 75 to 87 years. Twenty studies were of prospective design with sample sizes ranging from 30 to 450 [17-36], and three were of retrospective design [37-39], one of which contained a large sample size of nearly 13,000 participants [37]. Publications came from different countries, including USA [17, 18, 35, 37-39], UK

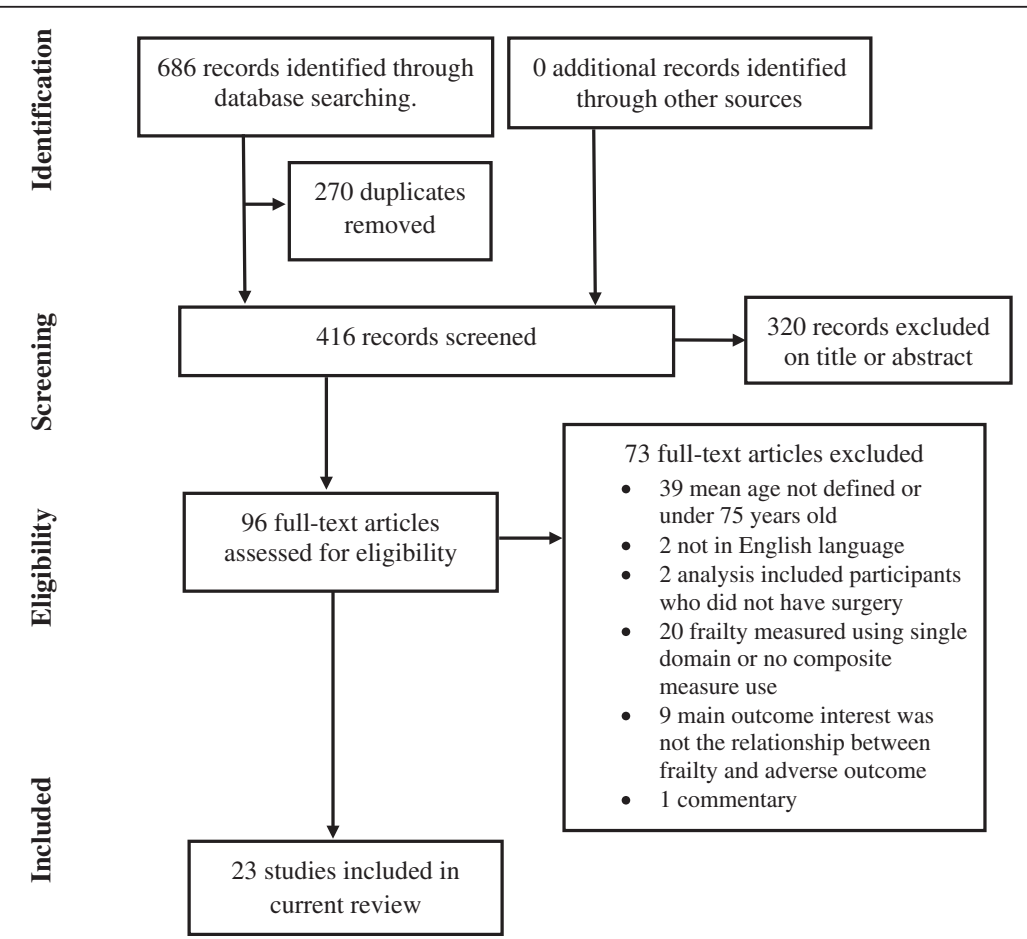

Fig. 1 PRISMA flow diagram for study selection 
[30, 32, 34, 36], Europe [19-28, 31], and Asia [29, 33]. The proportion of females ranged from $31 \%$ [34] to $83 \%$ [35]. Five studies did not report the gender distribution of the cohorts [22, 23, 29, 32, 38]. A meta-analysis was not conducted due to a lack of homogeneity of frailty measures and the diversity of surgical procedures.

Nine studies measured frailty in cardiac surgery $[17-24,39]$, six in oncological surgery (predominantly focusing on colorectal cancer) [25-29, 37], three in general surgery $[30,31,33]$, three in hip fracture surgery $[35,36,38]$ and two in vascular surgery [32, 34]. Sixteen articles involved participants undergoing elective surgery [17-29, 33, 37, 39], five involved those undergoing acute surgery $[30,31,35,36,38]$, while two included those undergoing both elective and acute surgery [32, 34]. Table 1, grouped by the type of surgery, describes the demographics, measurement of frailty and adverse outcome predicted by frailty for the selected studies.

\section{Study quality and risk of bias}

The EAI scores of the 23 studies ranged from 31 to 45, indicating they were in the upper two quartiles of study methodological quality. The EAI scores were in the in the second quartile for eight studies $[18,19,22-24,28,29,32]$ while the remainder 15 studies were in the first quartile $[17,20,21,25-27,30,31,33-39]$. There was a high level of agreement of quality assessment between the two independent reviewers. The most poorly reported items across all studies were: sample size calculation, adjustment for covariates and the report of losses to follow up. Study quality scores are incorporated into Table 2.

\section{Frailty instruments}

Of the 23 included studies, 21 different instruments were used to measure frailty. Variations of the Fried Criteria or instruments based on Comprehensive Geriatric Assessment (CGA), including the Frailty Index, were used in the majority of studies. Scales based on CGA are obtainable from patient interview as well as clinical notes without physical performance based measures, and were used in both acute and elective surgical cohorts. In contrast, the Fried frailty measure required physical performance-based tests, and was used exclusively in elective surgical cohorts. Four instruments, such as Multidimensional Frailty Score [33] and Comprehensive Assessment of Frailty [22-24], combined aspects of CGA with performance based tests (e.g. balance assessments, chair rise, stair climb) and medical investigations (e.g. blood test and respiratory function test). Details of measurement of frailty are presented in Table 1.

\section{Adverse outcomes predicted by frailty}

Table 2 shows the adverse outcomes associated with frailty, grouped by the quality of the studies. Short, intermediate and long term mortality were assessed by $16 \mathrm{pa}-$ pers. Of ten studies evaluating the relationship between frailty and 12 month mortality, all found a significant relationship with frailty $[18,19,21,23,24,32,33,37-39]$. Odds Ratios ranged between 1.1 and 4.97 for the frail patients compared with those who were non-frail $[18,21,23,24,38,39]$. This association was found regardless of the instruments used to measure frailty and irrespective of the type of surgery performed.

In the two papers that assessed long term mortality, frailty was associated with increased two year mortality with an Odds Ratio of 4.01 [38] and increased five year mortality with an Odds Ratio of 3.6 [27]. The association between frailty and 90 day mortality was evaluated in two studies [30, 37]. One found a significant association with an Odds Ratio of 10.4 [37] while the other did not find a significant association [30]. Thirty day mortality was evaluated in six studies [21, 22, 26, 30,31, 36]; all but one [30] found a significant association, with Odds Ratios ranging between 1.4 and 8.33 [21, 26, 31]. This latter study included only a small proportion $(31 \%, n=$ 105) of patients who underwent surgery [30].

Post-operative complications, as graded by the Clavian-Dindo severity classification [40] or predefined by the authors, were evaluated in nine papers [17, 18, 25, 29, 31, 33-35, 39]. Frailty was associated with increased post-operative complications in four studies with Odds Ratios ranging from 1.5 to $4.8[18,25$, $29,31]$. The remaining five studies reported no significant association [17, 33-35, 39]. The definitions used for post-operative complications in these 10 studies were heterogeneous. Conditions pre-specified in the studies which counted as a post-operative complication included cardiac complications (namely myocardial infarction, heart failure, arrhythmia), pulmonary embolism, pneumonia, wound infection, major bleeding, renal failure, delirium, unplanned return to theatre and unplanned intensive care unit admission.

Specific items of post-operative complications were also examined by several studies. An association between frailty and major cardiac and cerebral adverse events (MACCE) was reported by one of the three studies evaluating this outcome $[19,21,23]$. One study explored the association between frailty and delirium and did not find a significant association [35]. Of two studies evaluating frailty and readmission rate, one study found a significant association [32] while the other did not [30]. One study showed a significant association between frailty and the need for resuscitation [23].

Of the six studies that included prolonged length of stay as an outcome, an association with frailty was found 
Table 1 Study demographics grouped by type of surgery

\begin{tabular}{|c|c|c|c|c|c|}
\hline Author & $\begin{array}{l}\text { Sample size } \\
\text { Country of origin } \\
\text { Mean or median age } \\
\% \text { female } \\
\text { Study design }\end{array}$ & Type of surgery & Frailty measure & Adverse outcome predicted by frailty & $\begin{array}{l}\text { Association between frailty and } \\
\text { adverse outcomes }\end{array}$ \\
\hline \multicolumn{6}{|l|}{ Cardiac } \\
\hline Afilalo, J et al. [17] $]^{a}$ & $\begin{array}{l}152 \\
\text { USA, } \\
\text { Canada } \\
\text { Mean age } 75.9 \\
34 \% \text { female } \\
\text { Prospective cohort study }\end{array}$ & $\begin{array}{l}\text { Cardiac surgery } \\
\text { (Elective) }\end{array}$ & $\begin{array}{l}\text { Fried criteria (or Cardiovascular } \\
\text { Health Study frailty scale) } \\
\text { Modified CHS frailty scale } \\
\text { Fried + cognitive impairment + } \\
\text { depressed mood } \\
\text { 4-item MSSA frailty scale } \\
\text { gait speed, handgrip strength, } \\
\text { inactivity, cognitive impairment } \\
\text { Gait speed }\end{array}$ & $\begin{array}{l}\text { Composite end point of post- } \\
\text { operative mortality or major } \\
\text { morbidity }\end{array}$ & $\begin{array}{l}\text { Fried criteria, non-sig } \\
\text { Modified CHS frailty scale, non-sig } \\
4 \text { item MSSA frailty scale, non-sig } \\
\text { Gait speed, OR } 2.63(p<0.05)\end{array}$ \\
\hline Green, P et al. [39] ${ }^{a}$ & $\begin{array}{l}244 \\
\text { USA } \\
\text { Median age, \%female } \\
\text { - frail } 87.1,53 \% \\
\text { - non-frail } 85.4,45 \% \\
\text { Post-hoc analysis of } \\
\text { PARTNER trial }\end{array}$ & $\begin{array}{l}\text { Transcatheter Aortic } \\
\text { Valve Replacement } \\
\text { (TAVR) } \\
\text { (Elective) }\end{array}$ & $\begin{array}{l}\text { Fried criteria condensed into } 4 \\
\text { domains } \\
\text { gait speed, grip strength, serum } \\
\text { albumin, Katz index of } A D L \\
\text { Frail } \geq 6 / 12\end{array}$ & $\begin{array}{l}\text { 1) Adverse clinical events at } 30 \text { days } \\
\text { 2) } 1 \text { year mortality } \\
\text { 3) Poor outcome (composite mortality } \\
\& \text { QoL assessed by KCCQ-OS) } \\
\text { a) } 6 \text { months } \\
\text { b) } 1 \text { year }\end{array}$ & $\begin{array}{l}\text { Adjusted for covariates } \\
\text { 1) non-sig } \\
\text { 2) OR } 2.5(p=0.0002) \\
\text { 3) } \\
\text { a) OR } 2.21(p=0.03) \\
\text { b) OR } 2.4(p=0.02)\end{array}$ \\
\hline Green, P. et al. [18] $]^{b}$ & $\begin{array}{l}159 \\
\text { USA } \\
\text { Mean age } 86 \\
50 \% \text { female } \\
\text { Prospective cohort study }\end{array}$ & $\begin{array}{l}\text { Transcatheter aortic valve } \\
\text { replacement, (TAVR) } \\
\text { (Elective) }\end{array}$ & $\begin{array}{l}\text { Fried criteria condensed into } 4 \\
\text { domains } \\
\text { gait speed, grip strength, serum } \\
\text { albumin, Katz index of } A D L \\
\text { Frail }>5 / 12\end{array}$ & $\begin{array}{l}\text { 1) } 1 \text { year mortality } \\
\text { 2) LOS } \\
\text { 3) Procedural outcomes (any of } \\
\text { major bleeding event, major vascular } \\
\text { complications, stroke, acute kidney } \\
\text { injury, } 30 \text { day mortality) }\end{array}$ & $\begin{array}{l}\text { Adjusted for covariates } \\
\text { 1) OR } 3.5(p=0.006) \\
\text { 2) } 9 \text { vs } 6 \text { days }(p=0.004) \\
\text { 3) OR } 2.2(p=0.04) \text { for major bleeding } \\
\text { but not other adverse outcomes }\end{array}$ \\
\hline Kamga, M et al. [19] ${ }^{b}$ & $\begin{array}{l}30 \\
\text { Belgium } \\
\text { Mean age } 86 \\
47 \% \text { female } \\
\text { Prospective cohort study }\end{array}$ & $\begin{array}{l}\text { TAVI } \\
\text { (Elective) }\end{array}$ & $\begin{array}{l}\text { Score Hospitalier d'Evaluation du } \\
\text { Risque de Perte d'Autonomie } \\
\text { (SHERPA-risk of functional decline) } \\
\text { score } \\
\text { MMSE, age, perceived poor health, fall } \\
\text { in the last year, number of iADL } \\
\text { independently performed before } \\
\text { admission } \\
\text { Identification of Seniors at Risk (ISAR) } \\
\text { score } \\
>3 \text { medications, self reported memory } \\
\text { problems, sensory problems, hospital } \\
\text { admission within the last } 6 \text { months, } \\
\text { increased need for help at home }\end{array}$ & $\begin{array}{l}\text { 1) } 1 \text { year mortality } \\
\text { 2) Major cardiac and cerebral } \\
\text { adverse events (MACCE) }\end{array}$ & $\begin{array}{l}\text { Adjusted for covariates } \\
\text { 1) SHERPA HR2.74 for every } 1 \text { point } \\
\text { increase in score } \\
(p=0.004) \\
\text { ISAR non-sig } \\
\text { 2) SHERPA non-sig } \\
\text { ISAR non-sig }\end{array}$ \\
\hline $\begin{array}{l}\text { Schoenenberger, } \\
\text { A.W. et al. [20] }]^{\mathrm{a}}\end{array}$ & $\begin{array}{l}119 \\
\text { Switzerland } \\
\text { Mean age } 83.4 \\
55.5 \% \text { female } \\
\text { Prospective cohort study }\end{array}$ & $\begin{array}{l}\text { TAVI } \\
\text { (Elective) }\end{array}$ & $\begin{array}{l}\text { Mini Mental State Exam, Mini } \\
\text { Nutritional Assessment, TUG, BADL, } \\
\text { IADL, pre-clinical mobility disability } \\
\text { Frail } \geq 3\end{array}$ & $\begin{array}{l}\text { 1) Functional decline (BADL } \downarrow \geq 1 \\
\text { point) } \\
\text { 2) Functional decline or death } \\
\text { among all participants at } 6 \text { months }\end{array}$ & $\begin{array}{l}\text { Univariate } \\
\text { 1) OR } 3.31(p=0.02) \\
\text { 2) OR } 4.46(p=0.001)\end{array}$ \\
\hline
\end{tabular}


Table 1 Study demographics grouped by type of surgery (Continued)

\begin{tabular}{|c|c|c|c|c|c|}
\hline Stortecky, S. et al. [21] ${ }^{a}$ & $\begin{array}{l}100 \\
\text { Switzerland } \\
\text { Mean age } 83.7 \\
60 \% \text { female } \\
\text { Prospective cohort study }\end{array}$ & $\begin{array}{l}\text { TAVI } \\
\text { (Elective) }\end{array}$ & $\begin{array}{l}\text { Mini Mental State Exam, Mini } \\
\text { Nutritional Assessment, TUG, BADL, } \\
\text { IADL, pre-clinical mobility disability } \\
\text { Frail } \geq 3\end{array}$ & $\begin{array}{l}\text { 1) } 30 \text { day MACCE } \\
\text { 2) } 30 \text { day mortality } \\
\text { 3) } 1 \text { year MACCE } \\
\text { 4) } 1 \text {-year mortality }\end{array}$ & $\begin{array}{l}\text { Univariate analysis } \\
\text { 1) OR } 4.78(p=0.05) \\
\text { 2) OR } 8.33(p=0.03) \\
\text { 3) OR } 4.89(p=0.003) \\
\text { 4) OR } 3.68(p=0.02)\end{array}$ \\
\hline $\begin{array}{l}\text { Sundermann } \mathrm{S} \text {, } \\
\text { et al. }[22]^{\mathrm{b}}\end{array}$ & $\begin{array}{l}400 \\
\text { Germany } \\
\text { Mean age } 80.3 \\
\% \text { female not reported } \\
\text { Prospective cohort study }\end{array}$ & $\begin{array}{l}\text { Cardiac surgery } \\
\text { (Elective) }\end{array}$ & $\begin{array}{l}\text { Comprehensive Assessment of Frailty } \\
\text { Fried minus unintentional weight loss, } \\
\text { plus balance assessment, albumin, } \\
\text { creatinine, brain natriuretic peptide, } \\
\text { FEV1 and Clinical Frailty Scale } \\
\text { moderately frail }=11-25 \text { points } \\
\text { severely frail = } 26-35 \text { points }\end{array}$ & 30 day mortality & $\begin{array}{l}\text { Severely frail vs non frail } \\
21.7 \% \text { vs } 3.6 \% \\
A \cup C=0.71 \text { on logistic regression }\end{array}$ \\
\hline $\begin{array}{l}\text { Sundermann } \mathrm{S} \text {, } \\
\text { et al. }[23]^{\mathrm{b}}\end{array}$ & $\begin{array}{l}213 \\
\text { Germany } \\
\text { Mean age } 80.1 \% \text { female } \\
\text { not reported } \\
\text { Prospective cohort study }\end{array}$ & Cardiac surgery (Elective) & $\begin{array}{l}\text { CAF } \\
\text { FORECAST (Frailty predicts death } \\
\text { One year after Elective Cardiac } \\
\text { Surgery Tests) }\end{array}$ & $\begin{array}{l}\text { 1) } 1 \text { year mortality } \\
\text { 2) Requirement for resuscitation } \\
\text { 3) ICU stay } \\
\text { 4) MACCE } \\
\text { 1) } 1 \text { year mortality }\end{array}$ & $\begin{array}{l}\text { Adjusted for EuroSCORE } \\
\text { 1) OR } 1.097(p=0.001) \\
\text { AUC } 0.70 \\
\text { Frail vs non frail } \\
\text { 2) } 16 \% \text { vs } 2 \%(p<0.05) \\
\text { 3) non-sig } \\
\text { 4) non-sig } \\
\text { 1) FORECAST AUC } 0.76\end{array}$ \\
\hline $\begin{array}{l}\text { Sundermann } \mathrm{S} \text {, } \\
\text { et al. [24] }\end{array}$ & $\begin{array}{l}450 \\
\text { Germany } \\
\text { Mean age } 79 \\
50 \% \text { female } \\
\text { Prospective cohort study }\end{array}$ & Cardiac surgery (Elective) & $\begin{array}{l}\text { CAF } \\
\text { FORECAST } \\
\text { chair rise test, subjective weakness on } \\
\text { questionnaire, stair climbing, Clinical } \\
\text { Frail Scale and serum creatinine. }\end{array}$ & 1 year mortality & $\begin{array}{l}\text { Adjusted for age } \\
\text { CAF OR } 1.091(p<0.001) \\
\text { FORECAST OR } 1.265(p<0.001)\end{array}$ \\
\hline \multicolumn{6}{|l|}{ Oncologic } \\
\hline $\begin{array}{l}\text { Kristjansson S.R. } \\
\text { et al. [25] }\end{array}$ & $\begin{array}{l}178 \\
\text { Norway } \\
\text { Mean age } 79.63 \\
57 \% \text { female } \\
\text { Prospective cohort study }\end{array}$ & $\begin{array}{l}\text { Colorectal cancer surgery } \\
\text { (Elective) }\end{array}$ & $\begin{array}{l}\text { Balducci Frailty Criteria from CGA } \\
\text { Cumulative Illness Rating Scale (CIRS), } \\
\text { pADL, iADL, polypharmacy, MNA, } \\
\text { MMSE, and GDS }\end{array}$ & $\begin{array}{l}30 \text { day post-operative complications } \\
\text { (Clavian-Dindo grading) }\end{array}$ & $\begin{array}{l}\text { Adjusted for covariates } \\
\text { OR } 3.13 \text { (95\% Cl 1.65-5.92) }\end{array}$ \\
\hline $\begin{array}{l}\text { Kristjansson S.R. } \\
\text { et al. }[26]^{\mathrm{a}}\end{array}$ & $\begin{array}{l}176 \\
\text { Norway } \\
\text { Mean age } 80 \\
57 \% \text { female } \\
\text { Prospective longitudinal } \\
\text { study }\end{array}$ & $\begin{array}{l}\text { Cancer surgery } \\
\text { (Elective) }\end{array}$ & $\begin{array}{l}\text { Balducci Frailty Criteria from CGA } \\
\text { Modified Fried criteria }\end{array}$ & 30 day mortality & $\begin{array}{l}\text { Adjusted for cancer stage and age } \\
\text { Balducci OR } 3.39(p<0.001) \\
\text { Modified Fried OR } 2.67(p=0.029)\end{array}$ \\
\hline $\begin{array}{l}\text { Neuman, H.B. } \\
\text { et al. [37] }{ }^{\mathrm{a}}\end{array}$ & $\begin{array}{l}12,979 \\
\text { USA } \\
\text { Mean age } 84.4 \\
61.4 \% \text { female } \\
\text { Retrospective analysis of } \\
\text { Surveillance, Epidemiology } \\
\text { and End Results(SEER)- } \\
\text { Medicare database }\end{array}$ & $\begin{array}{l}\text { Colectomy for stage I to } \\
\text { III colon cancer } \\
\text { (Elective) }\end{array}$ & $\begin{array}{l}11 \text { item frailty measure defined by } \\
\text { the John Hopkins Adjusted Clinical } \\
\text { Group case-mix system } \\
\text { Difficulty walking, weight loss, frequent } \\
\text { falls, malnutrition, impaired vision, } \\
\text { decubitus ulcer, incontinence (plus } 4 \\
\text { additional unnamed conditions) } \\
\text { Frail } \geq 1 / 11\end{array}$ & $\begin{array}{l}\text { 1) } 90 \text { day survival } \\
\text { 2) } 1 \text {-year survival }\end{array}$ & $\begin{array}{l}\text { Adjusted for covariates } \\
\text { 1) OR } 10.4(p<0.001) \\
\text { 2) OR } 8.4(p<0.001)\end{array}$ \\
\hline
\end{tabular}

Requirement for resuscitation -

Frail vs non frai

$16 \%$ vs $2 \%(p<0.05)$

$1(p<0.001)$

chair rise test, subjective weakness on

questionnaire, stair climbing, Clinical

Frail Scale and serum creatinine.

Balducci Frailty Criteria from CGA

Cumulative Illness Rating Scale (CIRS), PADL, iADL, polypharmacy, MNA MMSE, and GDS

Balducci Frailty Criteria from CGA

Mean age 80

$57 \%$ female

study

the John Hopkins Adjusted Clinica

ix system

l unnamed conditions) 
Table 1 Study demographics grouped by type of surgery (Continued)

\begin{tabular}{|c|c|c|c|c|c|}
\hline $\begin{array}{l}\text { Ommundsen, N. } \\
\text { et al. [27] }\end{array}$ & $\begin{array}{l}178 \\
\text { Norway } \\
\text { Mean age } 80 \\
57 \% \text { female } \\
\text { Prospective cohort study }\end{array}$ & $\begin{array}{l}\text { Colorectal cancer surgery } \\
\text { (Elective) }\end{array}$ & Balducci Frailty Criteria from CGA & 5 year mortality & $\begin{array}{l}\text { Multivariate adjusted for TNM stage } \\
\text { and sex } \\
\text { OR } 3.6(p<0.001)\end{array}$ \\
\hline Ronning, B. et al. $[28]^{b}$ & $\begin{array}{l}84 \\
\text { Norway } \\
\text { Median age } 82 \\
59 \% \text { female } \\
\text { Prospective cohort study }\end{array}$ & $\begin{array}{l}\text { Colorectal cancer surgery } \\
\text { (Elective) }\end{array}$ & Balducci Frailty Criteria from CGA & $\begin{array}{l}\text { Post-operative functional status } \\
\text { 1) Barthel Index } \downarrow \\
\text { 2) NEADL } \downarrow \\
\text { 3) TUG } \uparrow \\
\text { 4) Grip strength } \downarrow\end{array}$ & $\begin{array}{l}\text { Logistic regression (95\% Cl) } \\
\text { 1) non-sig } \\
\text { 2) non-sig } \\
\text { 3) non-sig } \\
\text { 4) non-sig }\end{array}$ \\
\hline Tan, K-Y et al. [29] ${ }^{\mathrm{b}}$ & $\begin{array}{l}83 \\
\text { Singapore and Japan } \\
\text { Mean age } 81.5 \% \text { female } \\
\text { not reported } \\
\text { Prospective cohort study }\end{array}$ & $\begin{array}{l}\text { Colorectal cancer } \\
\text { (Elective) }\end{array}$ & Fried criteria & $\begin{array}{l}\text { Postop complications (Clavien- } \\
\text { Dindo } \geq \| \text { ) }\end{array}$ & $\begin{array}{l}\text { Bivariate analysis } \\
\text { OR } 4.08(p=0.006)\end{array}$ \\
\hline \multicolumn{6}{|l|}{ General/abdominal } \\
\hline Hewitt, J. et al. [30] ${ }^{a}$ & $\begin{array}{l}325 \\
\text { UK } \\
\text { Mean age } 77.6 \\
57 \% \text { female } \\
\text { Prospective cohort study }\end{array}$ & $\begin{array}{l}\text { General surgical patients } \\
\text { (Acute) } \\
\text { - only } 31 \% \text { underwent } \\
\text { surgery }\end{array}$ & $\begin{array}{l}\text { Clinical Frailty Scale } \\
7 \text { frailty levels based on visual } \\
\text { observation combined with an } \\
\text { abbreviated review of medical records } \\
\text { Frail is } \geq 5\end{array}$ & $\begin{array}{l}\text { 1) } 30 \text { day mortality } \\
\text { 2) } 90 \text { day mortality } \\
\text { 3) LOS } \\
\text { 4) } 30 \text { day hospital readmission }\end{array}$ & $\begin{array}{l}\text { Adjusted for age and polypharmacy, } \\
\text { frail vs non frail } \\
\text { 1) non-sig } \\
\text { 2) non-sig } \\
\text { 3) } 19 \text { vs } 7 \text { days }(p=0.02) \\
\text { 4) non-sig }\end{array}$ \\
\hline Kenig, J et al. [31] ${ }^{\mathrm{a}}$ & $\begin{array}{l}184 \\
\text { Poland } \\
\text { Mean age } 76.9 \\
53 \% \text { female } \\
\text { Prospective cohort study }\end{array}$ & $\begin{array}{l}\text { Abdominal surgery } \\
\text { (Acute) }\end{array}$ & $\begin{array}{l}\text { Vulnerable Elder Survey (VES) } \\
\text { age, self-rated health, limitation in } \\
\text { physical function and functional } \\
\text { disabilities } \\
\text { Triage Risk Screening Tool (TRST) } \\
\text { cognitive impairment, difficulty } \\
\text { walking/transferring/recent falls, } \geq 5 \\
\text { medications, ED use in previous } \\
30 \text { days or hospitalization in previous } \\
90 \text { days, lives alone and/or no available } \\
\text { caregiver, geriatric syndrome } \\
\text { G8 } \\
7 \text { items from the Mini Nutritional } \\
\text { Assessment (MNA) questionnaire and } \\
\text { age } \\
\text { Groningen Frailty Indicator (GFI) } \\
\text { ADLs, sensory impairment, nutrition, } \\
\text { polypharmacy, cognitive impairment, } \\
\text { psychosocial wellbeing and } \\
\text { subjective physical fitness } \\
\text { Rockwood's brief clinical instrument } \\
\text { to classify frailty (4 frailty levels) } \\
\text { Balducci Frailty Criteria }\end{array}$ & $\begin{array}{l}\text { 1) } 30 \text { day post-operative complica- } \\
\text { tions (Clavian-Dindo grading) } \\
\text { 2) } 30 \text { day mortality }\end{array}$ & $\begin{array}{l}\text { Adjusted for covariates } \\
\text { 1) VES: OR } 2.4(p<0.05) \\
\text { TRST: non-sig } \\
\text { G8: OR } 1.5(p<0.05) \\
\text { GFI: OR } 1.5(p<0.05) \\
\text { Rockwood: non-sig } \\
\text { Balducci: OR } 1.7(p<0.05) \\
\text { 2) VES: OR } 2.4(p<0.05) \\
\text { TRST: non-sig } \\
\text { G8: OR } 1.8(p<0.05) \\
\text { GFI: OR 1.4 }(p<0.05) \\
\text { Rockwood: non-sig } \\
\text { Balducci: OR } 1.4(p<0.05)\end{array}$ \\
\hline Kim, S et al. [33] ${ }^{a}$ & $\begin{array}{l}275 \\
\text { Korea } \\
\text { Mean age,\% female }\end{array}$ & $\begin{array}{l}\text { Intermediate or high risk } \\
\text { general surgery } \\
\text { (Elective) }\end{array}$ & $\begin{array}{l}\text { Multidimensional Frailty Score (MFS) } \\
\text { Malignant disease, Charleston } \\
\text { comorbidity Index, Albumin, ADLs, }\end{array}$ & $\begin{array}{l}\text { 1) } 1 \text { year mortality } \\
\text { 2) Discharge to residential care } \\
\text { 3) Postoperative complications }\end{array}$ & $\begin{array}{l}\text { Adjusted for covariates, for every } 1 \\
\text { point increase in MFS } \\
\text { 1) OR } 2.05(p<0.001)\end{array}$ \\
\hline
\end{tabular}


Table 1 Study demographics grouped by type of surgery (Continued)

\begin{tabular}{|c|c|c|c|c|c|}
\hline & $\begin{array}{l}\text { - survivors } 75.2,46 \% \\
\text { - deceased } 77.6,32 \% \\
\text { Prospective cohort study }\end{array}$ & & $\begin{array}{l}\text { IADLs, dementia, risk of delirium, } \\
\text { malnutrition, mid-arm circumference } \\
\text { Low risk } \leq 5 \\
\text { High risk }>5\end{array}$ & 4) LOS (median) & $\begin{array}{l}\text { 2) } \mathrm{OR} 1.42(p=0.01) \\
\text { 3) non-sig } \\
\text { 4) } 14 \text { vs } 9 \text { days for high vs low risk } \\
\text { group }(p<0.001)\end{array}$ \\
\hline \multicolumn{6}{|l|}{ Vascular } \\
\hline $\begin{array}{l}\text { Ambler, G.K. } \\
\text { et al. [32] }\end{array}$ & $\begin{array}{l}410 \\
\text { UK } \\
\text { Median age } 77 \% \\
\text { female not reported } \\
\text { Prospective cohort study }\end{array}$ & $\begin{array}{l}\text { Vascular surgery (Elective } \\
\text { and Acute) }\end{array}$ & $\begin{array}{l}\text { Addenbrooke's Vascular Frailty Score } \\
\text { (AVFS; } 6 \text { items, score } 0-6 \text { ) } \\
\text { Not independently mobile on } \\
\text { admission, depression, polypharmacy } \\
\text { on admission ( }>8 \text { medications), } \\
\text { anaemia, Waterlow score }>13 \text { on } \\
\text { admission, emergency admission }\end{array}$ & $\begin{array}{l}\text { 1) } 1 \text { year mortality } \\
\text { 2) Readmission-free survival } \\
\text { 3) Discharge to residential care } \\
\text { 3) Prolonged LOS }\end{array}$ & $\begin{array}{l}\text { Univariate; most vs least frail } \\
\text { 1) } 58 \% \text { vs } 0 \% \text {, AUC } 0.83 \\
\text { 2) } 0 \% \text { vs } 68 \%(p<0.001) \text {, AUC } 0.71 \\
\text { 3) AUC } 0.78 \\
\text { 4) AUC } 0.74\end{array}$ \\
\hline $\begin{array}{l}\text { Partridge, J.S.L. } \\
\text { et al. [34] }{ }^{\mathrm{a}}\end{array}$ & $\begin{array}{l}125 \\
\text { UK } \\
\text { Mean age } 76.3 \\
31 \% \text { female } \\
\text { Prospective observational } \\
\text { study }\end{array}$ & $\begin{array}{l}\text { Vascular surgery } \\
\text { (Elective and Acute) }\end{array}$ & $\begin{array}{l}\text { Edmonton Frail Scale (EFS) } \\
\text { cognitive impairment, dependence in } \\
\text { iADL, recent burden of illnesses, self- } \\
\text { perceived health, depression, weight } \\
\text { loss, medication issues, incontinence, } \\
\text { inadequate social support and } \\
\text { mobility difficulties. } \\
\text { Frail is }>7 / 18\end{array}$ & $\begin{array}{l}\text { 1) Composite measure post- } \\
\text { operative complications } \\
\text { 2) Composite measure adverse } \\
\text { functional outcomes } \\
\text { 3) LOS } \geq 12 \text { days }\end{array}$ & $\begin{array}{l}\text { Multivariate, adjusted for significant } \\
\text { baseline associations and age } \\
\text { 1) non-sig } \\
\text { 2) non-sig } \\
\text { 3) non-sig }\end{array}$ \\
\hline \multicolumn{6}{|l|}{ Hip fracture } \\
\hline Kistler, E et al. [35] & $\begin{array}{l}35 \\
\text { USA } \\
\text { Mean age } 86 \\
83 \% \text { female } \\
\text { Prospective cohort study }\end{array}$ & $\begin{array}{l}\text { Hip fracture surgery } \\
\text { (Acute) }\end{array}$ & Modified Fried Criteria & $\begin{array}{l}\text { 1) Post-operative complications } \\
\text { 2) Delirium } \\
\text { 3) LOS } \\
\text { 4) Time to surgery }\end{array}$ & $\begin{array}{l}\text { Frail vs Non-frail } \\
\text { 1) non-sig } \\
\text { 2) non-sig } \\
\text { 3) } 7.3 \text { vs } 4.1 \quad(p=0.038) \\
\text { 4) non-sig }\end{array}$ \\
\hline Krishnan, M et al. [36] & $\begin{array}{l}178 \\
\text { UK } \\
\text { Mean age } 81 \\
73.5 \% \text { female } \\
\text { Prospective cohort study }\end{array}$ & $\begin{array}{l}\text { Hip fracture surgery } \\
\text { (Acute) }\end{array}$ & FI (51 items) & $\begin{array}{l}\text { 1) } 30 \text {-day mortality } \\
\text { 2) Inpatient mortality } \\
\text { 3) LOS-failure to return home by } \\
30 \text { days }\end{array}$ & $\begin{array}{l}\text { Frail vs Non-frail } \\
\text { 1) } 17.2 \% \text { vs } 0 \%(p<0.001) \\
\text { 2) } 28.1 \% \text { vs } 0 \%(p<0.001) \\
\text { 3) AUC } 0.82\end{array}$ \\
\hline Patel K.V. et al. [38] & $\begin{array}{l}218 \\
\text { USA } \\
\text { Mean age } 81.2 \% \\
\text { female not reported } \\
\text { Retrospective chart review }\end{array}$ & $\begin{array}{l}\text { Hip fracture } \\
\text { (Acute) }\end{array}$ & Modified FI (19 items) & $\begin{array}{l}1 \text { year mortality } \\
2 \text {-year mortality }\end{array}$ & $\begin{array}{l}\text { OR } 4.97(p<0.001) \\
\text { OR } 4.01(p<0.001)\end{array}$ \\
\hline
\end{tabular}

andicates quartile 1 in the quality assessment

LOS length of stay, MACCE major cardiac \& cerebral adverse events, non-sig no statistically significant association, AUC area under the ROC curve for prediction of adverse outcomes 
Table 2 Adverse outcome associated with frailty, grouped by the quality of studies

\begin{tabular}{|c|c|c|c|c|c|c|c|c|c|c|c|}
\hline \multirow[t]{2}{*}{ Outcome } & \multicolumn{11}{|c|}{ Number of studies } \\
\hline & & 1 & 2 & 3 & 4 & 5 & 6 & 7 & 8 & 9 & 10 \\
\hline \multicolumn{12}{|l|}{ Mortality } \\
\hline 1 year Mortality & Quality [ref] & Q1 [21] & Q1 [33] & Q1 [39] & Q1 [37] & Q1 [38] & Q2 [18] & Q2 [19] & Q2 [23] & Q2 [24] & Q2 [32] \\
\hline$n=10$ & N sample & 100 & 275 & 244 & 12979 & 218 & 159 & 30 & 213 & 450 & 410 \\
\hline 2 Year Mortality & Quality [ref] & Q1 [38] & & & & & & & & & \\
\hline$n=1$ & N sample & 218 & & & & & & & & & \\
\hline 5 year Mortality & Quality [ref] & Q1 [27] & & & & & & & & & \\
\hline$n=1$ & N sample & 178 & & & & & & & & & \\
\hline 30 Day Mortality & Quality [ref] & Q1 [21] & Q1 [26] & Q1 [31] & Q1 [36] & Q2 [22] & Q1 [30] & & & & \\
\hline$n=6$ & N sample & 100 & 176 & 184 & 178 & 400 & 325 & & & & \\
\hline 90 Day Mortality & Quality [ref] & Q1 [37] & Q1 [30] & & & & & & & & \\
\hline$n=2$ & N sample & 12979 & 325 & & & & & & & & \\
\hline \multicolumn{12}{|c|}{ Post-Operative Complications } \\
\hline $\begin{array}{l}\text { Non-routine } \\
\text { recovery }\end{array}$ & Quality [ref] & Q1 [25] & Q1 [31] & Q2 [18] & Q2 [29] & Q1 [17] & Q1 [33] & Q1 [34] & Q1 [35] & Q1 [39] & \\
\hline$n=10$ & N sample & 178 & 184 & 159 & 83 & 152 & 275 & 125 & 35 & 244 & \\
\hline $\begin{array}{l}\text { Need for } \\
\text { resuscitation }\end{array}$ & Quality [ref] & Q2 [23] & & & & & & & & & \\
\hline$n=1$ & N sample & 213 & & & & & & & & & \\
\hline Delirium & Quality [ref] & Q1 [35] & & & & & & & & & \\
\hline$n=1$ & N sample & 35 & & & & & & & & & \\
\hline MACCE & Quality [ref] & Q1 [21] & Q2 [23] & Q2 [19] & & & & & & & \\
\hline$n=3$ & N sample & 100 & 213 & 30 & & & & & & & \\
\hline \multicolumn{12}{|l|}{ Discharge } \\
\hline Length of stay & Quality [ref] & Q1 [36] & Q1 [35] & Q1 [30] & Q2 [32] & Q2 [18] & Q1 [34] & & & & \\
\hline$n=6$ & N sample & 178 & 35 & 325 & 410 & 159 & 125 & & & & \\
\hline $\begin{array}{l}\text { Discharge to } \\
\text { Institution }\end{array}$ & Quality [ref] & Q1 [33] & Q2 [32] & & & & & & & & \\
\hline$n=3$ & N sample & 275 & 410 & & & & & & & & \\
\hline $\begin{array}{l}\text { Functional } \\
\text { Decline }\end{array}$ & Quality [ref] & Q1 [34] & & & & & & & & & \\
\hline$n=1$ & N sample & 125 & & & & & & & & & \\
\hline \multicolumn{12}{|l|}{ Post-Discharge } \\
\hline $\begin{array}{l}\text { Readmission } \\
\text { rate: } 1 \text { year }\end{array}$ & Quality [ref] & Q2 [32] & Q1 [30] & & & & & & & & \\
\hline$n=2$ & N sample & 410 & 325 & & & & & & & & \\
\hline $\begin{array}{l}\text { Functional } \\
\text { Decline }\end{array}$ & Quality [ref] & Q1 [20] & Q2 [28] & & & & & & & & \\
\hline$n=2$ & N sample & $\begin{array}{l}119 \\
\text { at } \\
6 \text { months }\end{array}$ & $\begin{array}{l}84 \\
16-28 \\
\text { months }\end{array}$ & & & & & & & & \\
\hline $\begin{array}{l}\text { Quality of Life: } \\
6 \text { months, } 1 \text { year }\end{array}$ & Quality [ref] & Q1 [39] & & & & & & & & & \\
\hline$n=1$ & N sample & 244 & & & & & & & & & \\
\hline
\end{tabular}


in five [18, 30, 32, 35, 36]. Three studies evaluated functional decline as an outcome, of which only one found a significant association [20]. Discharge to a residential care facility was found to be associated with frailty by both studies in which this outcome was evaluated [32, 33]. Quality of life was evaluated in one study and frailty was associated with the composite poor outcome of mortality or poorer quality of life [39].

Based on quality, quantity and consistency of the included studies, there is evidence for an association between frailty and adverse postoperative outcomes. Although cohort studies are lower on the hierarchy of evidence than randomised controlled trials, it is acknowledged that the cohort study design is entirely appropriate for investigating this particular research question. The literature search identified 23 studies that met the inclusion criteria and 15 of those were in the upper quartile of quality assessment, indicating the majority were methodologically sound. The consistency was evidenced by the finding that 20 of the included studies found evidence of an association between frailty and at least one adverse outcome.

\section{Discussion}

The reviewed studies consistently found that in patients aged over 75 years, frailty was associated with increased mortality, post-operative complications, prolonged length of stay and discharge to residential care facility. The strongest evidence of association was between frailty and 30 day mortality. The association was consistent across different frailty instruments and regardless of the type of surgery performed.

Our findings are congruent with other reviews of frailty in surgical patients. Beggs et al. found eight out of 19 articles demonstrating frailty to be significantly associated with mortality and post-operative complications [41]. Other systematic reviews have concentrated on specific surgical subspecialties, namely oncologic surgery [42], cardiac surgery [43] and thoracic surgery [44]. They also found frailty to impact negatively on post-operative outcomes. Two other reviews written on cardiac surgery also identified frailty as a risk factor that provided important prognostic information in older adults needing surgical or transcatheter aortic valve replacement [45] and found that frailty increased the predictive power of conventional risk scores [46].

The strength of this review is that it is inclusive of all types of surgery, both elective and acute, and focuses on those over 75 years old. This review provided insight into how frailty is measured and how it correlates with adverse outcomes in the 'old-old' and the 'oldest old' surgical population. Our search was limited to English publications, so may have excluded relevant publications in other languages. Another limitation was that studies using single markers to determine frailty, such as measurement of muscle mass or gait speed, were excluded based on the consensus view of frailty being a multidimensional state of increased vulnerability. Finally, due to the differences in frailty instruments used and heterogeneity of the surgical patient population, meta-analysis could not be conducted, and the magnitude of the adverse impact of frailty on outcome could not be estimated.

There is evidence that frailty is associated with increased mortality and morbidity in the older surgical patients. As patients over 75 years old are presenting more commonly for surgery, frailty assessment may have considerable value as a tool for peri-operative assessment. However, for the value of frailty assessment to be realised, it must not only predict outcomes but also be easily incorporated into routine assessment or created from existing information, without placing further resource burden on clinical staff and the patient. Once established, such a tool may offer a valuable addition to the risk assessment of older persons undergoing surgery, alongside the standard surgical and anaesthetic assessment tools. With the increasing focus on patient centred care, the ongoing development of frailty assessment has the potential to improve how well patients can be informed by their surgeons and anaesthetists prior to their procedures, thus enhancing informed consent. The clinical utility, time taken to make frailty assessments and the ease of use of most of the tools in the 23 included studies were not reported, which would be useful in assisting clinicians to decide which tool to adopt into clinical practice.

This review found several important gaps in the current literature. Frailty in acute surgical patients is under-studied. Only 7 out of 23 studies assessed acute surgical patients and all of them used scales based on comprehensive geriatric assessment to measure frailty. Reliance on performance based tests may be impractical in the acute surgical patients. More research into how frailty impacts on surgical patients in the acute setting and how best to measure frailty in acute surgical patients is needed. An instrument which is robust and valid for measuring frailty in elective patients in a surgical preadmission clinic may not be applicable to the acute patients. Despite the need to find a unified tool for measuring frailty, it is possible that different frailty tools are best suited for different acuity and type of surgical patients. Furthermore, these instruments need to be timeefficient and suitable for application at the bedside by staff who are not geriatricians.

Mortality and post-operative complications are the most commonly studied and reported outcomes in the 23 articles reviewed. Quality of life post-surgery was assessed in only one out of the 23 studies; similarly, 
functional decline and discharge to a care facility were only evaluated in three and two studies respectively. The association between frailty and functional outcome, discharge destination, and quality of life after surgery warrants further research. Factors and outcomes important to the individual elderly patient undergoing surgery must also be considered when performing pre-operative assessment, such as the consideration of premorbid status and return to the premorbid level of function.

\section{Conclusion}

Frailty is consistently found is to be associated with adverse outcomes after surgery. In the 23 articles reviewed, the strongest evidence lies in the association with increased 30 day, 90 day and 1 year mortality, postoperative complications and length of stay. This highlights the importance of detecting frailty in perioperative assessment. The possibility that different frailty tools may be best suited for different acuity and type of surgical patients is worth exploring. The association between frailty and return to pre-morbid function, discharge destination, and quality of life after surgery warrants further research.

\section{Acknowledgements}

Nil.

\section{Funding}

This research was partially funded by the Princess Alexandra Research Support Scheme to support a Fellowship in Clinical Research for first author.

\section{Availability of data and materials}

This paper is a systematic review. Articles selected for this review were referenced in bibliography. All data extracted from the selected studies were presented in the tables. There is no raw data to be made available.

\section{Authors' contributions}

$\mathrm{HL}$ and JW were responsible for the literature search, selection of articles for review, quality assessment of the articles and data extraction from the articles. HL was additionally responsible for writing the first draft of the paper and the synthesis of the results. NP and $\mathrm{RH}$ were responsible for the conception of the study design and aims and resolving any disagreement in the article assessment by HL and JW. All authors participated in the editing of the manuscript and have approved the final manuscript.

\section{Competing interests}

The authors declare that they have no competing interests.

\section{Consent for publication}

Not applicable.

\section{Ethics approval and consent to participate}

Not applicable.

Received: 21 April 2016 Accepted: 15 August 2016

Published online: 31 August 2016

\section{References}

1. Health \& Social Care Information Centre. Hospital Episode Statistics, Admitted Patient Care - England, 2014-15: Procedures and interventions. http://digital.nhs.uk/searchcatalogue?productid=19420\&q=Hospital+Episode +Statistics+Admitted+patient+care+2014\&topics=0\%2fHospital +care\&sort=Relevance\&size=10\&page=1\#top. Accessed 23 Aug 2016.
2. Health \& Social Care Information Centre. Hospital Episode Statistics, Admitted Patient Care - England, 2006-07: Main operations summaries. http://digital.nhs.uk/searchcatalogue?productid=92\&q=Hospital+Episode +Statistics+Admitted+patient+care+2006\&topics=0\%2fHospital +care\&sort=Relevance\&size=10\&page=1\#top. Accessed 23 Aug 2016.

3. Australian Institute of Health and Welfare. Australian hospital statistics 2012-13. In: Health services series; no 54. Canberra: Australian Institute of Health and Welfare; 2014.

4. Song X. Prevalence and 10-year outcomes of frailty in older adults in relation to deficit accumulation. J Am Geriatr Soc. 2010;58(4):681-7.

5. Rockwood K, Howlett SE, Macknight C, Beattie BL, Bergman H, Hebert R, et al. Prevalence, attributes, and outcomes of fitness and frailty in community-dwelling older adults: report from the Canadian study of health and aging. J Gerontol A Biol Sci Med Sci. 2004;59(12):1310-7.

6. Hubbard RE, Story DA. Does frailty lie in the eyes of the beholder? Heart Lung Circ. 2015;24(6):525-6.

7. de Vries NM, Staal JB, van Ravensberg CD, Hobbelen JS, Olde Rikkert MG, Nijhuis-van der Sanden MW. Outcome instruments to measure frailty: a systematic review. Ageing Res Rev. 2011;10(1):104-14.

8. Fried LP, Tangen CM, Walston J, Newman AB, Hirsch C, Gottdiener J, et al. Frailty in older adults: evidence for a phenotype. J Gerontol A Biol Sci Med Sci. 2001;56(3):M146-156.

9. Rockwood K, Song X, MacKnight C, Bergman H, Hogan DB, McDowell I, et al. A global clinical measure of fitness and frailty in elderly people. CMAJ. 2005;173(5):489-95.

10. Rockwood K, Mitnitski A. Frailty in relation to the accumulation of deficits. J Gerontol A Biol Sci Med Sci. 2007:62(7):722-7.

11. Singh I, Gallacher J, Davis K, Johansen A, Eeles E, Hubbard RE. Predictors of adverse outcomes on an acute geriatric rehabilitation ward. Age Ageing. 2012;41(2):242-6.

12. Arora RC, Rockwood K. Encyclopedia of Aging. 2002. from Encyclopedia. com: http://www.encyclopedia.com/doc/1G2-3402200400.html. Accessed 23 Aug 2016.

13. Morley JE, Vellas B, Abellan van Kan G, Anker SD, Bauer JM, Bernabei R, et al. Frailty consensus: a call to action. J Am Med Dir Assoc. 2013;14(6):392-7.

14. Gobbens RJ, Luijkx KG, Wijnen-Sponselee MT, Schols JM. In search of an integral conceptual definition of frailty: opinions of experts. J Am Med Dir Assoc. 2010;11(5):338-43.

15. Genaidy AM, Lemasters GK, Lockey J, Succop P, Deddens J, Sobeih T, et al. An epidemiological appraisal instrument - a tool for evaluation of epidemiological studies. Ergonomics. 2007;50(6):920-60.

16. West S, King V, Carey TS, Lohr KN, McKoy N, Sutton SF, et al. Systems to rate the strength of scientific evidence. Evidence Report/Technology Assessment No. 47 (Prepared by the Research Triangle Institute- University of North Carolina Evidence-based Practice Center under Contract No. 290-97-0011). Rockville, MD: Agency for Healthcare Research and Quality, 2002. AHRQ Publication No. 02-E016.

17. Afilalo J, Mottillo S, Eisenberg MJ, Alexander KP, Noiseux N, Perrault LP, et al. Addition of frailty and disability to cardiac surgery risk scores identifies elderly patients at high risk of mortality or major morbidity. Circ Cardiovasc Qual Outcomes. 2012:5(2):222-8.

18. Green P, Woglom AE, Genereux P, Daneault B, Paradis JM, Schnell S, et al. The impact of frailty status on survival after transcatheter aortic valve replacement in older adults with severe aortic stenosis: a single-center experience. JACC Cardiovasc Interv. 2012;5(9):974-81.

19. Kamga M, Boland B, Cornette P, Beeckmans M, De Meester C, Chenu P, et al. Impact of frailty scores on outcome of octogenarian patients undergoing transcatheter aortic valve implantation. Acta Cardiol. 2013;68(6):599-606.

20. Schoenenberger AW, Stortecky S, Neumann S, Moser A, Jüni P, Carrel T, et al. Predictors of functional decline in elderly patients undergoing transcatheter aortic valve implantation (TAVI). Eur Heart J. 2013;34(9):684-92.

21. Stortecky S, Schoenenberger AW, Moser A, Kalesan B, Juni P, Carrel T, et al. Evaluation of multidimensional geriatric assessment as a predictor of mortality and cardiovascular events after transcatheter aortic valve implantation. JACC Cardiovasc Interv. 2012;5(5):489-96.

22. Sundermann S, Dademasch A, Praetorius J, Kempfert J, Dewey T, Falk V, et al. Comprehensive assessment of frailty for elderly high-risk patients undergoing cardiac surgery. Eur J Cardiothorac Surg. 2011;39(1):33-7.

23. Sundermann S, Dademasch A, Rastan A, Praetorius J, Rodriguez $\mathrm{H}$, Walther $T$, et al. One-year follow-up of patients undergoing elective cardiac surgery assessed with the Comprehensive Assessment of Frailty test and its 
simplified form. Interact Cardiovasc Thorac Surg. 2011;13(2):119-23. discussion 123.

24. Sundermann SH, Dademasch A, Seifert B, Rodriguez Cetina Biefer H, Emmert MY, Walther T, et al. Frailty is a predictor of short- and mid-term mortality after elective cardiac surgery independently of age. Interact Cardiovasc Thorac Surg. 2014;18(5):580-5.

25. Kristjansson SR, Nesbakken A, Jordhoy MS, Skovlund E, Audisio RA, Johannessen $\mathrm{HO}$, et al. Comprehensive geriatric assessment can predict complications in elderly patients after elective surgery for colorectal cancer: a prospective observational cohort study. Crit Rev Oncol Hematol. 2010;76(3):208-17.

26. Kristjansson SR, Rønning B, Hurria A, Skovlund E, Jordhøy MS, Nesbakken A, et al. A comparison of two pre-operative frailty measures in older surgical cancer patients. J Geriatr Oncol. 2012;3(1):1-7.

27. Ommundsen N, Wyller TB, Nesbakken A, Jordhoy MS, Bakka A, Skovlund E, et al. Frailty is an independent predictor of survival in older patients with colorectal cancer. Oncologist. 2014;19(12):1268-75.

28. Rønning B, Wyller TB, Jordhøy MS, Nesbakken A, Bakka A, Seljeflot I, et al. Frailty indicators and functional status in older patients after colorectal cancer surgery. J Geriatr Oncol. 2014;5(1):26-32.

29. Tan KY, Kawamura YJ, Tokomitsu A, Tang T. Assessment for frailty is useful for predicting morbidity in elderly patients undergoing colorectal cancer resection whose comorbidities are already optimized. Am J Surg. 2012;204(2):139-43.

30. Hewitt J, Moug SJ, Middleton M, Chakrabarti M, Stechman MJ, McCarthy K. Prevalence of frailty and its association with mortality in general surgery. Am J Surg. 2015;209(2):254-9.

31. Kenig J, Zychiewicz B, Olszewska U, Barczynski M, Nowak W. Six screening instruments for frailty in older patients qualified for emergency abdominal surgery. Arch Gerontol Geriatr. 2015;61:437-42.

32. Ambler GK, Brooks DE, Al Zuhir N, Ali A, Gohel MS, Hayes PD, et al. Effect of frailty on short- and mid-term outcomes in vascular surgical patients. Br J Surg. 2015;102(6):638-45.

33. Kim SW, Han HS, Jung HW, Kim Kl, Hwang DW, Kang SB, et al. Multidimensional frailty score for the prediction of postoperative mortality risk. JAMA Surgery. 2014;149(7):633-40.

34. Partridge JSL, Fuller M, Harari D, Taylor PR, Martin FC, Dhesi JK. Frailty and poor functional status are common in arterial vascular surgical patients and affect postoperative outcomes. Int J Surg. 2015;18:57-63.

35. Kistler EA, Nicholas JA, Kates SL, Friedman SM. Frailty and Short-Term Outcomes in Patients With Hip Fracture. Geriatr Orthop Surg Rehabil. 2015;6(3):209-14.

36. Krishnan M, Beck S, Havelock W, Eeles E, Hubbard RE, Johansen A. Predicting outcome after hip fracture: using a frailty index to integrate comprehensive geriatric assessment results. Age Ageing. 2014;43(1):122-6.

37. Neuman HB, Weiss JM, Leverson G, O'Connor ES, Greenblatt DY, Loconte NK, et al. Predictors of short-term postoperative survival after elective colectomy in colon cancer patients $>/=80$ years of age. Ann Surg Oncol. 2013;20(5):1427-35.

38. Patel KV, Brennan KL, Brennan ML, Jupiter DC, Shar A, Davis ML. Association of a modified frailty index with mortality after femoral neck fracture in patients aged 60 years and older. Clin Orthop Relat Res. 2014:472(3):1010-7.

39. Green P, Arnold SV, Cohen DJ, Kirtane AJ, Kodali SK, Brown DL, et al. Relation of frailty to outcomes after transcatheter aortic valve replacement (from the PARTNER trial). Am J Cardiol. 2015;116(2):264-9.

40. Dindo D, Demartines N, Clavien PA. Classification of surgical complications: a new proposal with evaluation in a cohort of 6336 patients and results of a survey. Ann Surg. 2004;240(2):205-13.

41. Beggs T, Sepehri A, Szwajcer A, Tangri N, Arora RC. Frailty and perioperative outcomes: a narrative review. Can J Anaesth. 2015;62(2):143-57.

42. Feng MA, McMillan DT, Crowell K, Muss H, Nielsen ME, Smith AB. Geriatric assessment in surgical oncology: a systematic review. J Surg Res. 2015; 193(1):265-72.

43. Sepehri A, Beggs T, Hassan A, Rigatto C, Shaw-Daigle C, Tangri N, et al. The impact of frailty on outcomes after cardiac surgery: a systematic review. J Thorac Cardiovasc Surg. 2014;148(6):3110-7.

44. Dunne MJ, Abah U, Scarci M. Frailty assessment in thoracic surgery. Interact Cardiovasc Thorac Surg. 2014;18(5):667-70.

45. Wong CY, Green P, Williams M. Decision-making in transcatheter aortic valve replacement: the impact of frailty in older adults with aortic stenosis. Expert Rev Cardiovasc Ther. 2013;11(6):761-72.

46. Bagnall NM, Faiz O, Darzi A, Athanasiou T. What is the utility of preoperative frailty assessment for risk stratification in cardiac surgery? Interact Cardiovasc Thorac Surg. 2013;17(2):398-402.

\section{Submit your next manuscript to BioMed Central and we will help you at every step:}

- We accept pre-submission inquiries

- Our selector tool helps you to find the most relevant journal

- We provide round the clock customer support

- Convenient online submission

- Thorough peer review

- Inclusion in PubMed and all major indexing services

- Maximum visibility for your research

Submit your manuscript at www.biomedcentral.com/submit
C Biomed Central 